\title{
Impact of Emissivity and Reflectivity of the Object to Estimate Temperature Using Infrared Thermal Imaging Camera
}

\author{
Ž. Barbarić, A. Pavlović, V. Nikolić
}

\begin{abstract}
The calculation of the energy efficiency of buildings is carried out on the basis of the measurements (external and internal building temperature, emissivity of the outer and inner surfaces of the wall) and the appropriate construction parameters. The aim of this paper is to show the impact of changes of emissivity and reflectivity on the accuracy of the temperature of building materials and heat flux construction. The experiment was performed using Infrared Thermal Imaging Camera FLIR T 420. The samples are building materials- brick, fir, tin and some type of marble. Analysis of the generated images is done in FLIR Tools software package. We estimated equivalent temperature of objects as functions of emissivity end reflectivity non transparent objects.Obtained experimental results show that the equivalent temperature increases if emissivity decreases, and equivalent temperature increases if reflectivity of object increases.
\end{abstract}

Keywords: equivalent temperature, emissivity, reflectivity, infrared thermal imager (thermovision).

\section{Introduction}

This research was partially supported by Ministry of Science of Serbia, under the grant TR32023.

Energy efficiency programs in the construction industry are important for reducing the energy consumption of expensive imported raw materials as well as for the development of urban areas with preserved quality of human habitats and also for increasing the international competitiveness of the products and services of the Serbian economy [1].

The goal of energy efficiency in the construction is to be more rational in consumption of energy required for the construction-reconstruction, heating and utility supply of office space, and to permanently reduce the current significant losses in the distribution and use of

Manuscript received June 28, 2014; accepted December 4, 2014.

Ž. Barbarić, A. Pavlović,V. Nikolić are with the State University of Novi Pazar, Novi Pazar, Serbia; 
existing and new construction and infra structural facilities. The energy passport is based on measurements of infrared thermal imaging camera and heat transfer calculations. As it known, transfer heat has three components: conduction, convention and emittance. The emittance part of the heat may be estimate using Infrared thermal imager (thermovision).

The calculation of the energy efficiency of buildings is carried out on the basis of the measurements (external and internal building temperature, emissivity of the outer and inner surfaces of the wall) and the appropriate construction parameters. [1]

In Serbia, today, we consume 2-3 times more energy per square meter of housing or office space [1], it is clear the importance of development of energy efficiency in buildings, which should contribute to increasing energy efficiency and reducing energy consumption in the residential sector.

The aim of this paper is to show impact of changes of emissivity and reflectivity on the accuracy of the temperature of building materials and heat flux construction. In this experiment were used next buildings materials: brick, fir, tin and marble. The materials are from well- known manufacturers and their features are well known. For the measurements were used the following equipment: a digital contact sensor for measuring temperature, and thermal imaging camera FLIR T420, to generate infrared images. Generated images are analyzed in the software package Flir Tools + .

\section{Theoretical background}

As is known, the radiance from scene has three components: radiation of objects or background, atmospheric radiation and reflection of radiation of the Sun and stars, as well as reflection of man-made sources from the scene [2]. Schematic representation of mapping object plane xoy in the image plane $x_{1} o y_{1}$ and radiation components of scene are shown on Fig 1.

From Fig. 1 it can be seen that the object and surroundings are located in the field of view of thermal imaging camera, which are replicated in thermal image by integration of radiance of terrain by spatial viewing angle of thermal imaging camera. Fig. 1 schematically shows emitted and reflected radiance from elementary surface of terrain, with natural ground surface $\Delta x \Delta y$. Also, Fig. 1 shows the irradiance of the Sun, which falls on the ground and radiance of atmosphere, which is included in spatial viewing angle of the image sensor.

Spectral radiance $L_{\lambda}$ from elementary surface of object $\Delta x \Delta y$, (Fig. 1) is given in next the equation:

$$
L_{\lambda}=L_{\lambda}^{E}+L_{\lambda}^{R}=e_{\lambda} L_{\lambda}^{C T}(T)+\frac{1}{\pi} \rho_{\lambda} E_{\lambda}^{S}
$$

where are: $L_{\lambda}^{E}$-spectral emitted radiance, and $L_{\lambda}^{R}$ - spectral reflected radiance, $L_{\lambda}^{C T}$-spectral radiance of black body (Plank's law), $e_{\lambda}$-spectral emissivity of real body temperature $\mathrm{T}$, $\rho_{\lambda}$-spectral reflectivity, $E_{\lambda}^{S}$-spectral irradiance from the Sun (from other sources). 


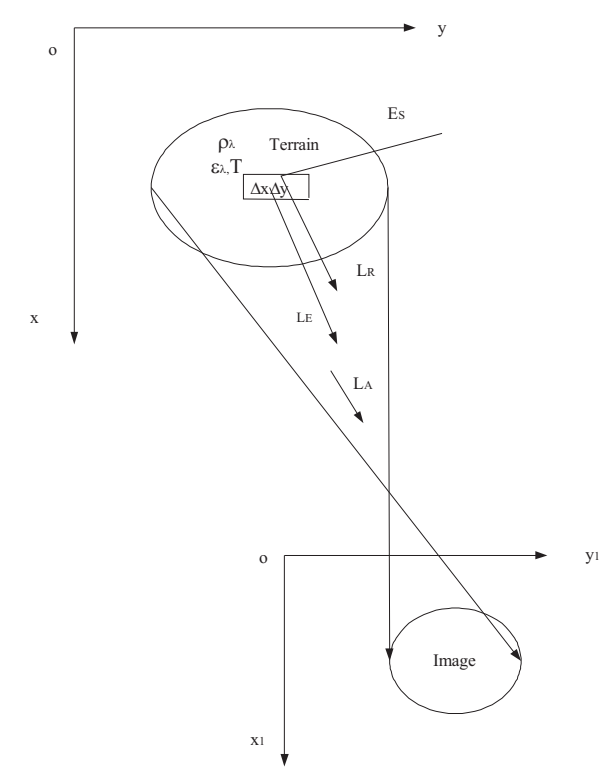

Fig. 1: Schematic representation radiation of scene and mapping the plane of object in the plane of image

Both components of spectral radiance from elementary surface of object $\Delta x \Delta y$ to the sensor (camera) passing through atmosphere, which has spectral transmission and additional radiate, so at the entrance of sensor, we have equivalent spectral radiance:

$$
L_{\lambda}^{E K}=\tau_{\lambda}\left(L_{\lambda}^{E}+L_{\lambda}^{R}\right)=\tau_{\lambda} L_{\lambda}+L_{\lambda}^{A}
$$

where are: $\tau_{\lambda}$ - spectral transsmitance of atmosphere, and $L_{\lambda}^{A}$ - spectral radiance of atmosphere.

Given spectral radiances (2) are function coordinates of the scene and time. Since the time of imaging is much less than second, and time constant scene is order of 100 seconds, the time dependence can be ignored. Also, at short distances, atmosphere is considered as ideal.

Optical sensor system mapping the distribution of radiance from elementary surfaces of the scene on the detector elements and form a image of the scene, as shown in Fig. 1. This mapping scene in the imaging plane can be represented by convolution integral of spectral radiance and spectral optical transfer function, $O_{\lambda}$ of image sensor

$$
L_{\lambda}^{E S}\left(x_{1}, y_{1}\right)=\int_{-\infty}^{\infty} \int_{-\infty}^{\infty} L_{\lambda}^{E K}(x, y) O_{\lambda}\left(x_{1}-x, y_{1}-y\right) d x d y
$$

where are: $x, y$-coordinates on the scene, $x_{1}, y_{1}$-coordinates in image plane, and $L_{\lambda}^{E S}$ - equivalent spectral radiance mapped into image plane. 
The detector performs integration of mapped spectral radiance by the wavelength and transforms the incident radiation into a voltage signal, which is amplified andforms image signal or level of gray in

$$
I\left(x_{1}, y_{1}\right)=G \int_{\lambda_{1}}^{\lambda_{2}} \mathscr{R}_{\lambda} E_{\lambda}^{E S}\left(x_{1}, y_{1}\right) d \lambda
$$

Where are: G- constant of proportionality, $\Re_{\lambda}$ - spectral conversion factor of detector sensor. Equation (4) shows that the gray level of image depends on distribution of spectral radiance of scene, optical transfer function, and type of detector. In addition to gray level, in image analysis is often used contrast on image of scene.

The complexity of process of detection of objects in the image scene can be understood with additional statistical analysis of the relations (4). The fluctuation of gray levels in the image scene depends on fluctuations radiance of the scene and noise of detector sensor. The influence of fluctuations radiance of scene is dominant, and detection of objects in the forest background is very convenient.

\section{Equivalent temperature}

Thermovision receives the radiance from scene, as irradiance.The temperature is inversion function of irradiance

$$
E=\int_{\lambda_{1}}^{\lambda_{2}} \mathscr{R}_{\lambda}\left(e_{\lambda} L_{\lambda}^{C T}+\frac{1}{\pi} \rho_{\lambda} E_{\lambda}^{D T}\right) d \lambda
$$

where are: $L_{\lambda}^{C T}$ - the spectral radiance of black body (Planck's law), $e_{\lambda}$ - the spectral emissivity, $\rho_{\lambda}$ - the spectral reflectivity and $E_{\lambda}^{D T}$ - the spectral irradiance of other bodies.

Equivalent irradiance and gray level are functions of temperature, emissivity and reflectivity.Our aim is to estimate temperature distribution on the thermal infrared image.Estimation of temperature based on received irradiance from the scene, which is also a function of temperature, emissivity and reflectivity.

$$
E=F(T, e, \rho)
$$

Estimation of the temperature based on the spectral irradiance difference is given by

$$
\Delta E_{\lambda}=L_{\lambda}^{C T} \Delta e_{\lambda}+e_{\lambda} \Delta L_{\lambda}^{C T}+\frac{1}{\pi} E_{\lambda}^{D T} \Delta \lambda_{\lambda}+\frac{1}{\pi} \rho_{\lambda} \Delta E_{\lambda}^{D T}
$$

where, $\Delta L_{\lambda}^{C T} \cong \frac{\Delta T}{T} \frac{C_{2}}{\lambda T}$ is the spectral radiance difference of black body as function of temperature difference. 


\section{Experiment results}

\subsection{Experiment tools}

The aim of this paper is to show impact of changes of emissivity and reflectivity on the accuracy of the temperature of building materials and heat flux construction. In this experiment were used next buildings materials: brick, fir, tin and marble. The materials are from well- known manufacturers and their features are well known.For the measurements are used the following equipment: a digital contact sensor for measuring temperature, and thermal imaging camera FLIR T420, to generate infrared images. Generated images are analyzed in the software package Flir Tools +. [5]

\subsection{Estimation of emissivity}

Estimation of emissivity of materials is performed at room temperature. Temperature of every material is measured using digital contact sensor. By using the thermal imaging camera for the correction emissivity, the emissivity of the material was changed until is received a temperature equal to the temperature obtained with digital contact sensor. The conditions for measurements were: the temperature of ambient $\mathrm{T}_{A}=24^{0} \mathrm{C}$, the distance form object $\mathrm{d}=1 \mathrm{~m}$ and relatively humidity $\mathrm{r}=40 \%$. Using digital contact sensor, the next temperature values are obtained: $\mathrm{T}_{B}=25.9^{0} \mathrm{C}$ (brick), $\mathrm{T}_{F}=26^{0} \mathrm{C}$ (fir), $\mathrm{T}_{T}=25.9^{0}$ (tin) and $\mathrm{T}_{M}=25.8^{0} \mathrm{C}$ (marble). Using infrared thermal imaging camera, the next emissivity values are estimated: $e_{B}=0.81$ (brick), $e_{F}=0.84$ (fir), $e_{T}=0.92$ (tin) and $e_{M}=0.81$ (marble).

\subsection{Examples of temperature estimation}

In this experiment were performed the two different ways for temperature estimation:

a) the temperature estimation as function emissivity, 1

b) the temperature estimation as function emissivity and reflectivity.

The materials were used: fir, tin and marble.

Examples of this two different ways for temperature estimation are shown at the thermal images given in Figure 2, 3 and 4, and the temperature estimation are shown in Tables 1 to 6. The temperature estimation is performed using Infrared Thermal Imaging Camera with properly set emissivity.

From Tables 1 and 2, it can be see that maximum temperature is increased for $0.4^{0} \mathrm{C}$, minimum temperature is increased for $0.1^{0} \mathrm{C}$ and average temperature is increased for $0.2^{0} \mathrm{C}$.

From Tables 3 and 4, it can be see that maximum temperature is increased for $0.2^{\circ} \mathrm{C}$, minimum temperature is increased for $0.7^{0} C$ and average temperature is increased for $0.3^{0} C$. 


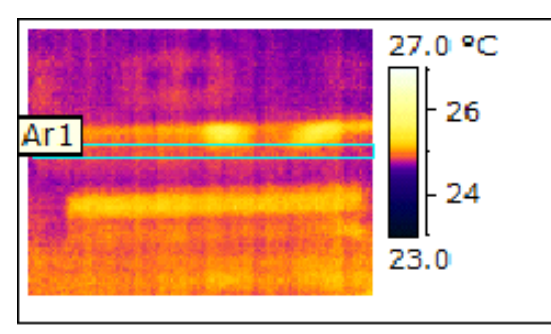

(a)

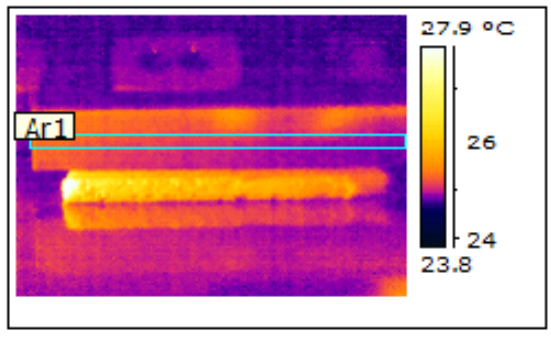

(b)

Fig. 2: a) Temperature estimation as function emissivity- fir (thermal image) b) Temperature estimation as function emissivity and reflectivity - fir (thermal image)

Table 1: Results of temperature estimation as function emissivity- fir

\begin{tabular}{|l|l|}
\hline Ar1 Max. Temperature & $25.2^{0} \mathrm{C}$ \\
\hline Ar1 Min. Temperature & $24.6^{0} \mathrm{C}$ \\
\hline Ar1 Average Temperature & $24.9^{0} \mathrm{C}$ \\
\hline
\end{tabular}

From Tables 5 and 6, it can be see that maximum temperature is increased for $0.2^{0} C$, minimum temperature is increased for $0.2^{\circ} \mathrm{C}$ and average temperature is increased for $0.3^{0} \mathrm{C}$.

Table 2: Results of temperature estimation as function emissivity and reflectivity- fir

\begin{tabular}{|l|l|}
\hline Ar1 Max. Temperature & $25.6^{0} \mathrm{C}$ \\
\hline Ar1 Min. Temperature & $24.7^{0} \mathrm{C}$ \\
\hline Ar1 Average Temperature & $25.1^{0} \mathrm{C}$ \\
\hline
\end{tabular}

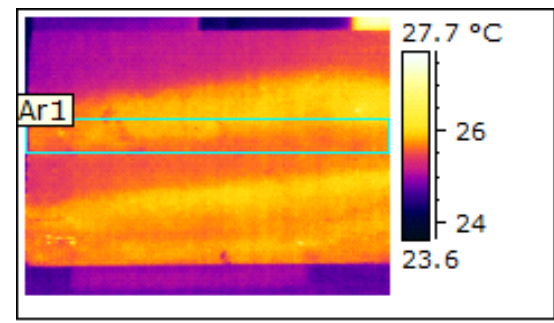

(a)

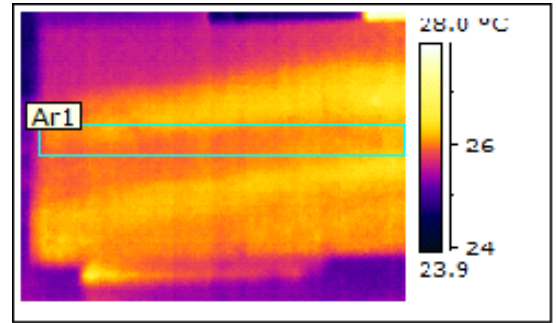

(b)

Fig. 3: a) Temperature estimation as function of emissivity - tin (thermal image) b) Temperature estimation as function emissivity and reflectivity- tin (thermal image) 
Table 3: Results of temperature estimation as function emissivity- tin

\begin{tabular}{|l|l|}
\hline Ar1 Max. Temperature & $26.2^{0} \mathrm{C}$ \\
\hline Ar1 Min. Temperature & $24.8^{0} \mathrm{C}$ \\
\hline Ar1 Average Temperature & $25.7^{0} \mathrm{C}$ \\
\hline
\end{tabular}

Table 4: Results of temperature estimation as function emissivity and reflectivity- tin

\begin{tabular}{|l|l|}
\hline Ar1 Max. Temperature & $26.4^{0} \mathrm{C}$ \\
\hline Ar1 Min. Temperature & $25.5^{0} \mathrm{C}$ \\
\hline Ar1 Average Temperature & $26^{0} \mathrm{C}$ \\
\hline
\end{tabular}

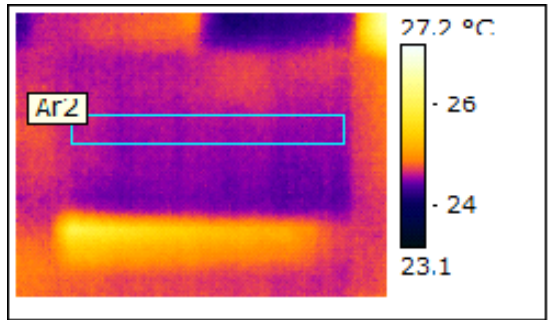

(a)

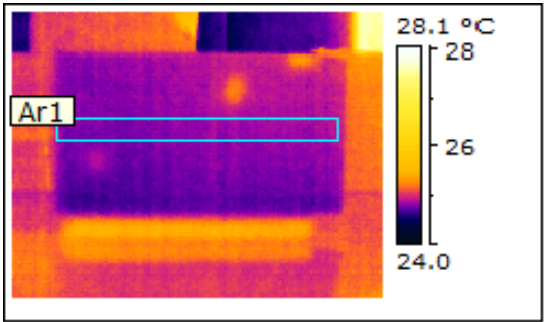

(b)

Fig. 4: a) Temperature estimation as function of emissivity - marble (thermal image) b) Temperature estimation as function emissivity and reflectivity- marble (thermal image)

Table 5: Results of temperature estimation as function emissivity- marble

\begin{tabular}{|l|l|}
\hline Ar1 Max. Temperature & $24.9^{0} \mathrm{C}$ \\
\hline Ar1 Min. Temperature & $24.4^{0} \mathrm{C}$ \\
\hline Ar1 Average Temperature & $24.5^{0} \mathrm{C}$ \\
\hline
\end{tabular}

Table 6: Results of temperature estimation as function emissivity and reflectivity- marble

\begin{tabular}{|l|l|}
\hline Ar1 Max. Temperature & $25.1^{\circ} \mathrm{C}$ \\
\hline Ar1 Min. Temperature & $24.6^{\circ} \mathrm{C}$ \\
\hline Ar1 Average Temperature & $24.8^{0} \mathrm{C}$ \\
\hline
\end{tabular}




\section{Conclusions}

In this paper, we present impact of changes of emissivity and reflectivity on the accuracy of the temperature of building materials and heat flux construction. For estimation energy efficiency of buildings, it can be used thermovision, which gives some information about temperature of object.Accuracy of the temperature estimation depends on emissivity and reflectivity of object.Obtained experimental results show that the equivalent temperature increases if emissivity decreases, and equivalent temperature increases if reflectivity of object increases.

\section{References}

[1] A. PAVlović, Z. BARBARIĆ, Analysis of Irregularity of Public Facilities by the Use of Fusion of TV and Thermal Images, 2nd International Conference CONTEMPORARY PROBLEMS OF MATHEMATICS, MECHANICS AND INFORMATICS (CPMMI 2012), State University of Novi Pazar, 2012.

[2] Ž. BARbarić, A. MARinČIĆ, G. Petrović, D. Milovanović, Thermal-image generation by line-scanning technique: a new computer model, Applied Optics, Vol. 33, No. 14, 1994, pp. 2883-2891.

[3] Driggers R.G., Cox P., Edwards T., Introduction to Infrared and Electro-Optical Systems, Artech House, Boston-London, 1999.

[4] M. Vollmer, K.-P. Mollmann, Infrared Thermal Imaging: Fundamentals, Research and Applications, WILEY-VCH Verlag GmbH, 2010.

[5] User's Manual, Flir Tool/Tools+, http://support.flir.com 